\title{
Targeting the CALR interactome in myeloproliferative neoplasms
}

\author{
Elodie Pronier, ${ }^{1,2}$ Paolo Cifani, ${ }^{3}$ Tiffany R. Merlinsky, ${ }^{1,2}$ Katharine Barr Berman, ${ }^{1,2}$ \\ Amritha Varshini Hanasoge Somasundara, ${ }^{1,2}$ Raajit K. Rampal, ${ }^{1,4}$ John LaCava, ${ }^{5}$ Karen E. Wei, ${ }^{1,2}$ \\ Friederike Pastore, ${ }^{1,2}$ Jesper L.V. Maag, ${ }^{2}$ Jane Park, ${ }^{2}$ Richard Koche, ${ }^{2}$ Alex Kentsis, ${ }^{3,6}$ \\ and Ross L. Levine, ${ }^{1,2,4,7}$ \\ ${ }^{1}$ Human Oncology and Pathogenesis Program, ${ }^{2}$ Center for Epigenetics Research, and ${ }^{3}$ Molecular Pharmacology Program, \\ Sloan Kettering Institute, New York, New York, USA. ${ }^{4}$ Leukemia Service, Department of Medicine, Memorial Sloan \\ Kettering Cancer Center, New York, New York, USA. 5Laboratory of Cellular and Structural Biology, The Rockefeller \\ University, New York, New York, USA. ${ }^{6}$ Department of Pediatrics, Weill Cornell Medical College of Cornell University and \\ Memorial Sloan Kettering Cancer Center, New York, New York, USA. 'Center for Hematologic Malignancies, Memorial Sloan \\ Kettering Cancer Center, New York, New York, USA.
}

Mutations in the ER chaperone calreticulin (CALR) are common in myeloproliferative neoplasm (MPN) patients, activate the thrombopoietin receptor (MPL), and mediate constitutive JAK/STAT signaling. The mechanisms by which CALR mutations cause myeloid transformation are incompletely defined. We used mass spectrometry proteomics to identify CALR-mutant interacting proteins. Mutant CALR caused mislocalization of binding partners and increased recruitment of FLI1, ERP57, and CALR to the MPL promoter to enhance transcription. Consistent with a critical role for CALR-mediated JAK/STAT activation, we confirmed the efficacy of JAK2 inhibition on CALR-mutant cells in vitro and in vivo. Due to the altered interactome induced by CALR mutations, we hypothesized that CALR-mutant MPNs may be vulnerable to disruption of aberrant CALR protein complexes. A synthetic peptide designed to competitively inhibit the carboxy terminal of CALR specifically abrogated MPL/ JAK/STAT signaling in cell lines and primary samples and improved the efficacy of JAK kinase inhibitors. These findings reveal what to our knowledge is a novel potential therapeutic approach for patients with CALR-mutant MPN.

Conflict of interest: RLL is on the supervisory board of QIAGEN and is a scientific advisor to Loxo Oncology, Imago Systems, C4 Therapeutics, and IsoPlexis. He receives research support from and consulted for Celgene and Roche, receives research support from Prelude Therapeutics, and has consulted for Novartis and Gilead Sciences. He has received honoraria from Lilly and Amgen for invited lectures. RKR has received consulting fees from Incyte, Celgene, Agios Pharmaceuticals, Apexx Oncology, and Jazz Pharmaceuticals, and has received research funding from Constellation Pharmaceuticals, Incyte, and Stemline Therapeutics.

License: Copyright 2018, American Society for Clinical Investigation.

Submitted: June 5, 2018 Accepted: September 19, 2018 Published: November 15, 2018

Reference information: JCI Insight. 2018;3(22):e122703. https://doi.org/10.1172/jci. insight.122703.

\section{Introduction}

Gene discovery studies have revealed that the majority of the remaining patients with $J A K 2 / M P L$-WT myeloproliferative neoplasms (MPNs) harbor somatic calreticulin $(C A L R)$ mutations $(1,2)$. The most common mutations are either 52-base pair deletions or 5-base pair insertions; these mutations result in expression of an altered C-terminal domain with loss of negative charge, impaired $\mathrm{Ca}^{++}$binding, and deprivation of the KDEL ER retention motif. A central role for mutant $C A L R$ in early MPN ontogeny has been demonstrated using in vivo and in vitro models: $C A L R$ mutations contribute to MPN development (3-6), are detectable in the long-term hematopoietic stem cell compartment $(1,2)$, and are associated with increased MPL/JAK/STAT signaling $(3,7,8)$. Here, using affinity chromatography coupled with mass spectrometry proteomics, we delineated the $C A L R$-mutant interactome and demonstrated that $C A L R$ mutations promote the formation of abnormal protein chaperone complexes, which alter their cellular localization and result in increased FLI1, ERP57, and CALR recruitment to the $M P L$ promoter. We also confirmed that $C A L R$-mutant cells are sensitive to JAK2 inhibition in vitro and in vivo. We then designed a synthetic peptide corresponding to the C-terminal sequence of WT CALR (C-Term peptide) and showed that this peptide inhibits MPL/JAK/STAT signaling in cell lines and primary cells expressing CALR mutants, which is further accentuated when combined with JAK inhibitor therapy. Our studies reveal important insights on the mechanisms by which $C A L R$ mutants promote transformation and define what to our knowledge is a novel therapeutic strategy specific for MPN patients harboring CALR mutations. 


\section{Results}

Mass spectrometry-based identification of CALR-mutant interactomes. To elucidate the functional effects of $C A L R$ mutations on hematopoietic cells, we engineered Ba/F3 cells to express WT or mutant CALR as fusion proteins with the maltose-binding protein (MBP), given its ability to maintain physiologic protein folding (9). Thus, we introduced empty vector (EV), MBP-tagged mutant CALR (deletion [DEL] or insertion [INS]), or MBP-tagged CALR WT cDNA into MPL-WT-Ba/F3 cells. We also generated a construct with the INS mutation followed by a stop codon (STOP) to create a truncation at the site of the mutation, such that the construct expressed the mutant protein without expression of the additional C-terminus, to fully determine the phenotypes specifically associated with the new C-terminal sequence (Supplemental Figure 1A; supplemental material available online with this article; https://doi.org/10.1172/jci.insight.122703DS1). To identify binding partners of WT and mutant CALR, we performed label-free quantitation (LFQ) of proteins purified using MBP affinity chromatography, followed by high-resolution nanoscale liquid chromatography and high-accuracy mass spectrometry of native extracts of MPL-WT-Ba/F3 cells expressing WT, DEL, INS, or STOP CALR compared with an IgG control (Figure 1, A-D, Supplemental Figure 1, B and $C$, and supplemental material). This identified a subset of proteins (12 bound to DEL CALR and 7 to INS CALR), which were significantly enriched in association with mutant CALR compared to WT CALR (Figure 1, A and B). As previously described $(10,11)$, we observed that total CALR protein levels were increased in cells harboring WT CALR but not mutant CALR, suggesting that the mutant forms of the protein are less stable than the WT protein (Supplemental Figure 1, D and E). To control for potential differences in purification efficiencies due to differences in expression, we normalized the LFQ of significantly impacted partners $(P<0.01)$ to the one of MBP (Figure 1, C and D, and Supplemental Figure 1F). Our results indicated that DEL CALR- and INS CALR-mutant forms were enriched for binding to proteins implicated in the activation of the unfolded protein response induced by ER stress (Hspa5/BiP, Hspa9/ Grp75, and Hspa8), proteins of the cytoskeleton (My19 and Aprc4), and ribosomal proteins (Rp17, Rsp23, and Rp111) (Figure 1C and Supplemental Figure 1F). In addition, we found that the CALR-DEL mutant exhibited increased copurification with the megakaryocyte transcription factor Fli1 (Figure 1, A and C). Furthermore, we noticed that the CALR-mutant proteins showed decreased binding affinities for Pdia3/ Erp57 and Msi2 compared with WT CALR (Figure 1D). Immunoprecipitation experiments, followed by Western blot analysis, in MPL-WT-Ba/F3 cells expressing mutant or WT CALR constructs or negative controls (EV and IgG), confirmed differential binding to BiP, My19, Grp75, Fli1, and Erp57 (Figure 1E). We also observed an association between MPL and both WT and mutant CALR (Supplemental Figure 1, $\mathrm{G}$ and $\mathrm{H}$, and supplemental material), as previously described $(3,7,12)$. Given that ectopic expression of mutant, but not WT, CALR confers IL-3 independence $(3,5)$ to MPL-WT-Ba/F3 cells, we assessed the interaction between mutant CALR and candidate interacting proteins in cytokine-free growth conditions. We observed increased Fli1 binding to INS CALR in the absence of IL-3, whereas we found similar binding between mutant CALR and BiP, My19, and Erp57 with or without cytokine supplementation (Supplemental Figure 1I). In all, these data demonstrate that $C A L R$ mutations promote the formation of abnormal protein chaperone complexes, with increased binding affinities to a subset of known CALR partners (e.g., $\mathrm{BiP}$ ) and, to our knowledge, previously undescribed interacting proteins (Fli1 and My19).

CALR mutants' binding to a subset of partners affects their cellular localization and MPL transcription. Although primarily localized in the ER, WT CALR has also been shown to be present in the nucleus, where it is involved in the nuclear import of glucocorticoid and steroid hormone receptors $(13,14)$. We hypothesized that $C A L R$ mutations affect its chaperone activity and result in changes in the cellular localization of a subset of its partners. We found an increase in protein expression levels of Myl9 and Fli1 in cytoplasmic fractions and elevated expression of BiP protein in the membrane/organelle compartment of CALR-mutant cells (Figure 2A). As expected, in CALR mutant-expressing cells, we observed increased Stat3 phosphorylation (pStat3) in the nuclear and chromatin fractions compared with control cells. Moreover, we found increased expression of Fli1 in chromatin extracts from CALR mutant-expressing cells (Figure 2B). We detected WT and mutant forms of CALR in the nucleus and in the chromatin fraction of MPL-WT-Ba/F3 cells (Figure 2B). Similar results were obtained by comparing cytoplasmic and nuclear protein extractions of mononuclear cells isolated from healthy donors (CTRL) and from patients with $C A L R$-mutated MPN (Supplemental Figure 2A). From these samples, we confirmed increased protein levels of BIP and MYL9 in cytoplasmic extracts of mononuclear cells isolated from patients with CALR-mutated MPN. We also detected elevated FLI1 protein expression in nuclear extracts of patients 

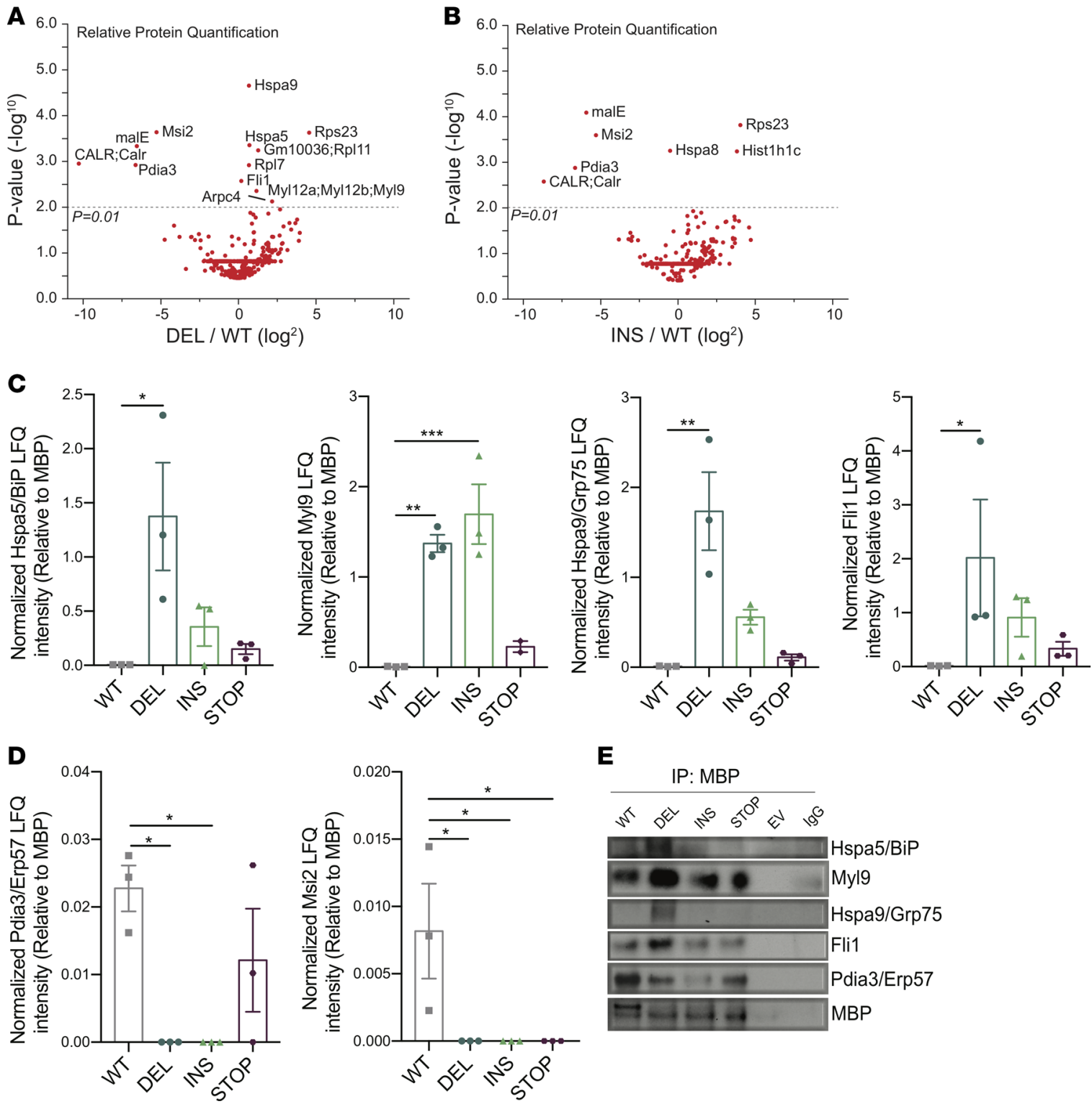

$\mathbf{E}$

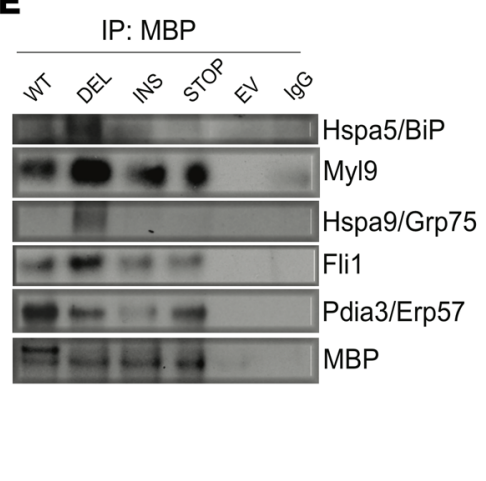

Figure 1. Mass spectrometry-based identification of CALR-mutant interactomes. Volcano plots showing the degrees of enrichment (ratio of LFQ protein intensities) in MPL-WT-Ba/F3 cells expressing (A) DEL CALR compared with WT CALR cells and (B) INS CALR compared with WT CALR cells. The $x$ axis depicts the fold change in protein levels and the $y$ axis the $-\log ^{10} P$ value. For each, 3 independent experiments were performed, and the data were analyzed with MaxQuant. Changes in partner's abundance are shown for each condition. Normalized LFQ intensities of a subset of candidate proteins to the MBP LFQ of each condition, for proteins that are (C) enriched (Hspa5/BiP, Myl9, Hspa9/Gp75, and Fli1) or (D) lost (Pdia3/ Erp57 and Msi2) in mutant cells compared with WT CALR-expressing cells. (E) Confirmation of differential binding between WT CALR and mutant proteins with a subset of targets by co-immunoprecipitation (Hspa5/BiP, Myl9, Hspa9/Grp75, Fli1 and Pdia3/Erp75) in MPL-WT-Ba/F3 cells. MBP was used as an immunoprecipitation efficacy control. In $\mathbf{C}$ and $\mathbf{D}$, mean values $\pm \mathrm{SEM}$ are represented. Statistical significance was assessed using 1-way ANOVA. ${ }^{*} P<0.05$, ${ }^{* *} P<0.01$, and ${ }^{* * *} P<0.001$. Blots are representative of 3 independent experiments.

with CALR-mutant MPN compared with controls (Figure 2C). These data suggest that WT and mutant CALR can function as a chaperone from the cytoplasm to the nucleus and that this can differentially modulate nuclear/chromatin recruitment of CALR-mutant versus WT interacting proteins.

Fli1 encodes an ETS transcription factor that can directly bind to the MPL promoter and induce its expression (15). We hypothesized that CALR-mediated chaperoning of Fli1 to the chromatin would lead to increased MPL transcription. We observed, in MPL-WT-Ba/F3; -UT-7 and c-Kit ${ }^{+}$cells expressing CALR mutants (DEL or INS), elevated mRNA expression of endogenous MPL compared with EV and WT controls $(P<0.0001)$ (Figure 2D and Supplemental Figure 2B). Previous studies have reported a role of Erp57 in Stat3 activation (16) and nuclear localization, suggesting an important role in transcriptional activation (17-19). 
A

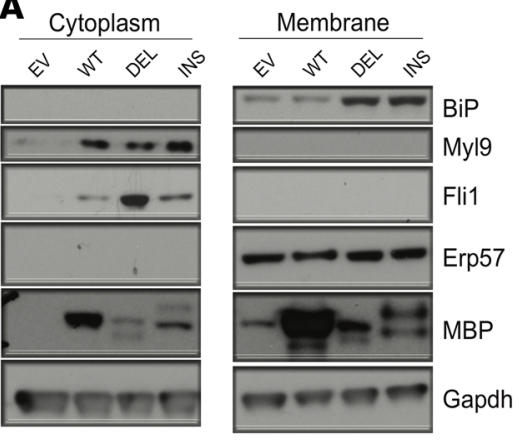

B

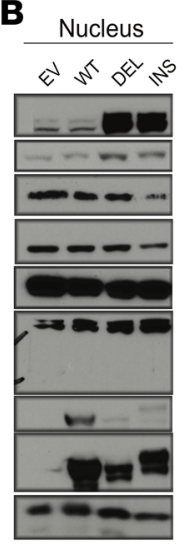

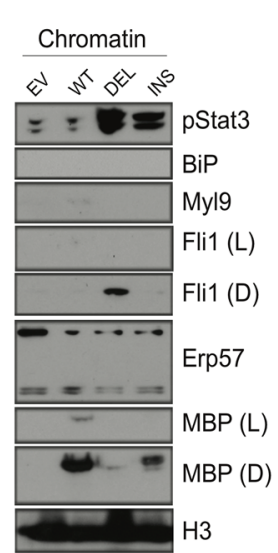

$\mathbf{F}$

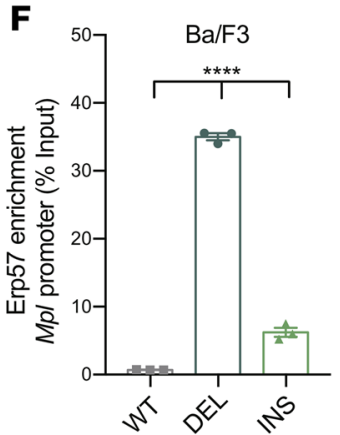

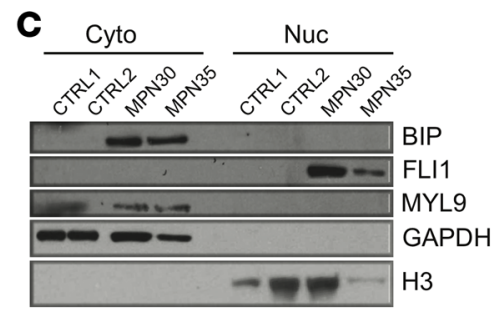

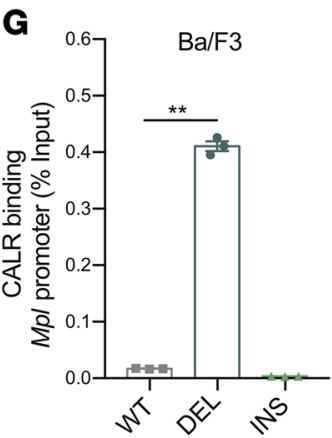

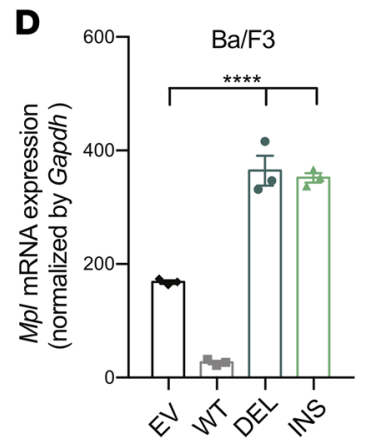
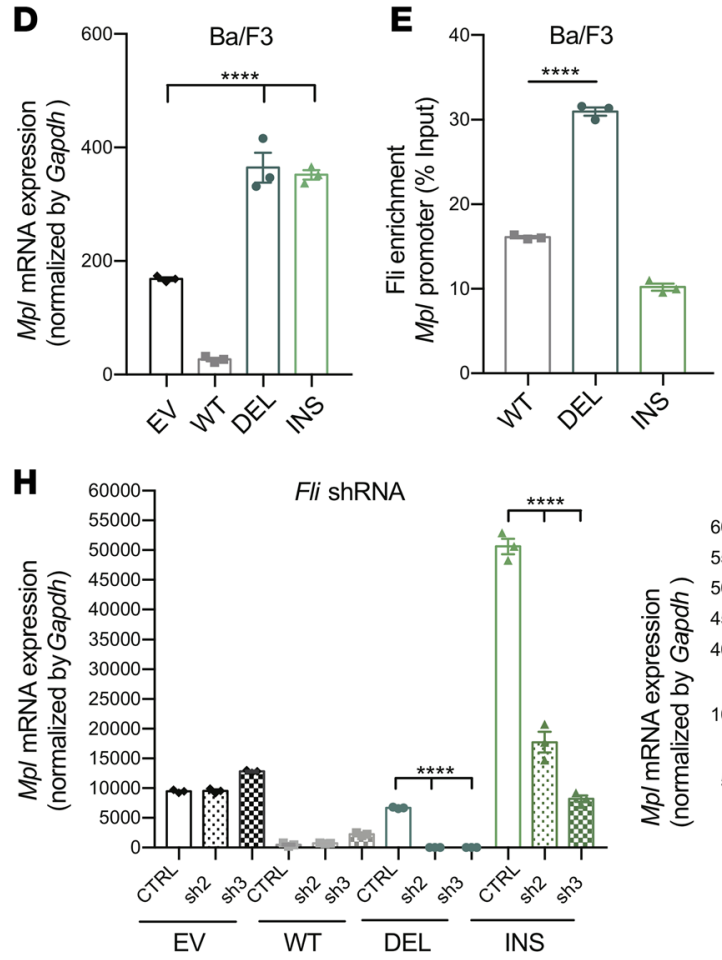

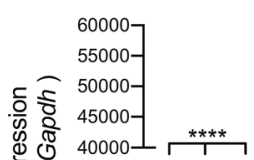

Erp57 shRNA

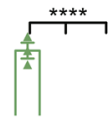

I

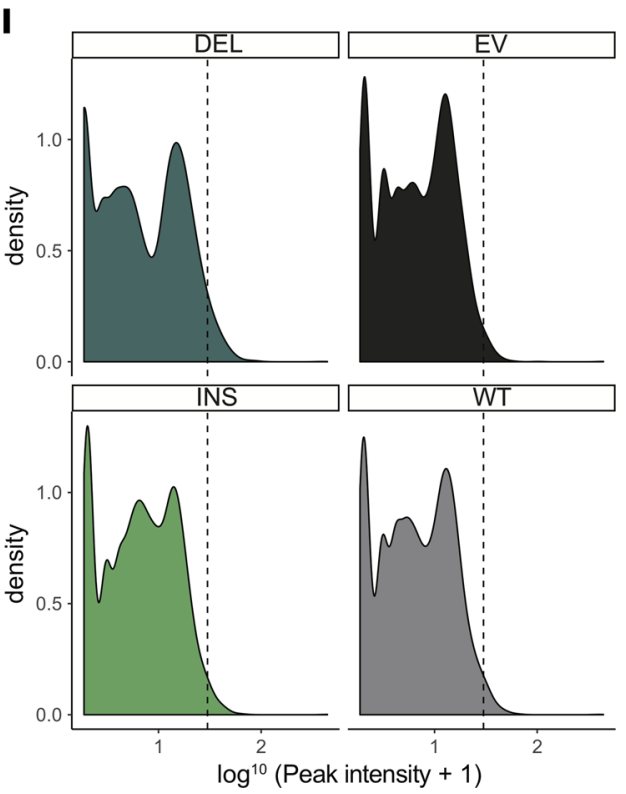

히을

迹

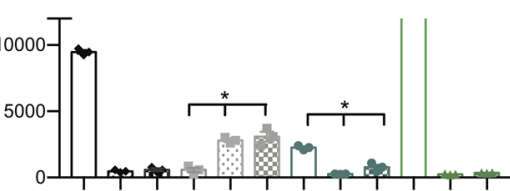

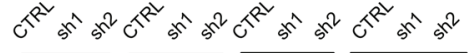
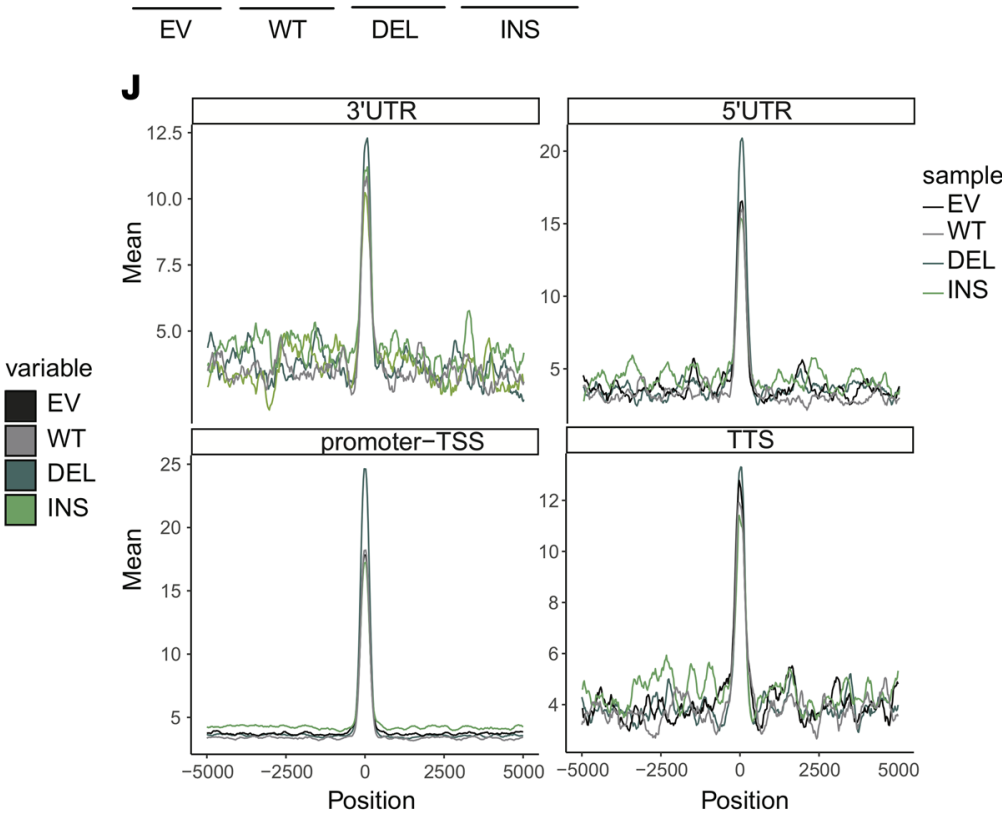
Figure 2. CALR mutants' binding to a subset of partners affects their cellular localization and influences MPL transcription. (A) Cytoplasm/membrane extracts and (B) nucleus/chromatin extracts from EV or WT CALR-, DEL CALR-, and INS CALR-expressing MPL-WT-Ba/F3 cells were used to detect, by Western blot analysis, cellular localization of CALR (MBP) and its partners: BiP, Myl9, Fli1, and Erp57. L, light exposure time; D, dark exposure time. (C) Cytoplasm (Cyto) and nuclear (Nuc) extracts from mononuclear cells isolated from healthy donors (CTRL) or patients with CALR-mutated MPN. (D) Quantitative reverse transcription PCR (qRT-PCR) analysis of MPL expression in MPL-WT-Ba/F3 cells expressing WT, DEL, or INS CALR compared with EV control. qRT-PCR analysis of ChIP assays showing (E) Fli1, (F) Erp57, and (G) CALR binding to the Mpl promoter in MPL-WT-Ba/F3 cells expressing WT, DEL, or INS CALR. Data are expressed as the percentage of pre-immunoprecipitation input for each sample and are representative of at least 3 independent immunoprecipitations. (H) qPCR analysis of $\mathrm{Mpl}$ in MPL-WT-Ba/F3 cells expressing EV or WT, DEL, or INS CALR transduced by Fli1 or Erp57 shRNA compared with Renilla controls (CTRL). (I) Signal distribution of Fli1 ChIP-seq peaks' intensity in EV or WT CALR-, DEL CALR-, or INS CALR-expressing cells. (J) Mean peak intensity of Fli1 peaks per the following genomic regions: 3'-UTR, 5'-UTR, promoter TSSs, and TSSs. TSS, transcription start site for MPL-WT-Ba/F3 cells expressing EV or WT, DEL, or INS CALR. In D-H, mean values \pm SEM are represented. Statistical significance was assessed using 1 -way ANOVA. ${ }^{*} P<0.05$, ${ }^{*} P<0.01$, and ${ }^{* * *} P<0.0001$. Blots are representative of 3 independent experiments. Gapdh or histone H3 was used as a loading control. Gapdh was used as a housekeeping gene ( $n=3$ in triplicate).

Moreover, CALR is known to act as an important modulator of the regulation of gene transcription by nuclear hormone receptors $(14,20)$. Based on these observations, we hypothesized that ERP57 and CALR could also directly bind to the MPL promoter in CALR-mutant cells to regulate its transcription. To investigate FLI1, ERP57, and CALR recruitment to the MPL promoter $(21,22)$, we performed ChIP analysis on MPL-WT-Ba/F3; -UT-7 and total bone marrow (BM) cells isolated from WT mice expressing WT or mutant CALR. Consistent with our data showing increased MPL mRNA expression, we detected significantly enriched recruitment of FLI1, ERP57, and CALR to the MPL promoter in CALR-mutant cells compared with WT CALR controls (Figure 2, E-G, and Supplemental Figure 2, C-E). Of note, differential recruitment of FLI1, ERP57, and CALR to the MPL promoter was seen with the different CALR mutations, which could be explained, in part, by differences in FLI1 expression levels or involvement of other unknown partners. To further confirm the direct impact of Fli1 and Erp57 on MPL expression, we transduced MPLWT-Ba/F3 cells expressing WT CALR, DEL CALR, INS CALR, or EV with either control or 2 independent Fli1- or Erp57-targeting shRNA vectors and confirmed significant knockdown efficiency by quantitative reverse transcription PCR (qRT-PCR) (Supplemental Figure 2F). Consistent with our hypothesis, we found that $M p l$ mRNA expression was significantly decreased in Fli1- or Erp57-reduced cells expressing DEL and INS CALR compared with control cells (Figure 2H). We also noticed lower $M p l$ expression associated with Erp57 silencing in control cells (EV), suggesting that Erp57 regulates the $M p l$ locus in the presence and absence of CALR mutations (Figure 2H). In accordance with these results, Fli1 shRNA- or Erp57 shRNAmediated silencing in cells expressing DEL or INS CALR reduced JAK/STAT signaling and attenuated the IL-3-independent growth of CALR mutant-expressing cells (Supplemental Figure 2, G and H). These results demonstrate that CALR mutants can regulate $M P L$ transcription by recruiting upstream regulators to the nucleus and inducing further activation of the $M P L$ promoter.

$C A L R$-DEL mutant influences the genome-wide recruitment of Fli1 to the chromatin. Given the enhanced Fli1 localization to chromatin in $C A L R$-mutated cells, we hypothesized that expression of $C A L R$ mutations could influence Fli1 occupancy genome wide. We performed Fli1 ChIP-sequencing (ChIP-seq) experiments in MPL-WT-Ba/F3 cells expressing EV or WT, DEL, or INS CALR. The peak-calling program MACS2 was applied to the Fli1 ChIP-seq and input sequence data to identify potential Fli1-occupied segments in all conditions. Strikingly, we observed an overall elevated intensity of signal in DEL CALR-expressing cells compared with the other conditions (versus EV $P=1.074^{-08}$, WT $P=8.38^{-10}$, versus INS $P=1.75^{-08}$; Figure 2I). Moreover, we found increased proportions of Fli1 peaks at promoters and transcription start sites (TSSs) and to a lesser extent at 3'-UTR regions and in intergenic and noncoding regions of DEL CALR-expressing cells compared with controls (Figure 2J and Supplemental Figure 3, A-C). Differences in the Fli1 cistrome were not observed in WT CALR- or INS CALR-expressing cells. Overall, these results show that type $1 C A L R$ mutant modulates Fli1 cellular localization and recruitment.

CALR-mutated cells are sensitive to JAK1/2 inhibitors in vitro and in vivo. Given the enhanced JAK/STAT activity in $C A L R$ mutant-transformed MPL-WT-Ba/F3 cells, we next evaluated the sensitivity of these cells to Jak2 inhibition in vitro and in vivo. The type I JAK2 inhibitor, ruxolitinib (Rux), is approved for polycythemia vera and primary myelofibrosis $(\mathrm{MF})$ and has been shown to decrease splenomegaly and constitutional symptoms in both diseases and to ameliorate disease in a $C A L R$-mutant mouse model $(6,23,24)$. We previously demonstrated that MPN cells can acquire an adaptive form of resistance that can be abrogated by type II inhibitors, such as CHZ868 (CHZ) $(25,26)$. Because it has been shown that $C A L R$-mutated patients were characterized by constitutive JAK2 activation, we tested the sensitivity of $C A L R$-mutant cells to type I and 
type II JAK2 inhibitors. CHZ868 potently inhibited the proliferation of CALR-mutated MPL-WT-Ba/F3 cells $\left(\mathrm{IC}_{50}\right.$ mean $=341 \mathrm{nM}$ for DEL and $434 \mathrm{nM}$ for INS, versus $696 \mathrm{nM}$ for WT) (Figure 3A, Supplemental Figure 4A, and supplemental material). CHZ868 treatment potently induced apoptosis in CALR-mutant cells, as reflected by caspase-3 activation (Figure 3B) and abrogated phosphorylation of Y1007/Y1008 in the JAK2 activation loop, consistent with a previously described type II mechanism of action in Jak2/Mpl-mutant cells (Figure 3C and ref. 26). We observed similar effects with the type I JAK2 inhibitor, Rux (Supplemental Figure $4, \mathrm{~A}-\mathrm{C}$ ). To assess the efficacy of $\mathrm{CHZ} 868$ at inhibiting Ba/F3 proliferation in vivo, we transplanted MPLWT-Ba/F3 cells expressing DEL or INS CALR mutations into sublethally irradiated BALB/c mice. We found a significant increase in survival in CHZ868-treated animals as compared with vehicle-treated mice $(P<0.01)$ (Figure 3D). In addition, CHZ868 therapy significantly reduced splenomegaly, hepatomegaly, WBC, and mutant allele burden, as reflected by a reduction in the proportion of $\mathrm{GFP}^{+}$cells in the $\mathrm{BM}$ and the spleen of treated animals $(P<0.0001)$ (Figure 3, E and F). These findings show that type II JAK2 inhibition efficiently inhibits $C A L R$-mutant cells in vivo and in vitro and demonstrate that JAK inhibitors represent a tractable approach to target $C A L R$-mutated MPN cells.

$C A L R$-mutant cells are sensitive to a peptide corresponding to the C-terminal sequence of the WT CALR. We next sought to delineate whether therapeutic approaches that altered the interaction between mutant CALR and candidate effectors could inhibit fitness and attenuate constitutive JAK/STAT signaling in CALR mutant cells. Based on differences between WT and mutated CALR with respect to their C-terminal sequence, we hypothesized that a peptide encoding for the C-terminal sequence of WT protein (C-Term) could competitively inhibit mutant CALR binding to interacting proteins and demonstrate therapeutic efficacy. We first validated our ability to achieve peptide delivery in MPL-WT-Ba/F3 cells by biotinylating the peptide (Bio-C-Term) and performing immunoprecipitation experiments using streptavidin with protein extracts isolated from cells at different time points after treatment (0 to 240 minutes). We detected peptide uptake in these cells for as long as 120 minutes after treatment (Supplemental Figure 4D). Because $C A L R$ mutants have been previously described as sufficient to transform cells to cytokine independence $(8,12)$, we hypothesized that treatment of MPL-WT-Ba/F3 cells with the peptide would reverse this phenotype and reduce IL-3-independent growth of $C A L R$ mutant-expressing cells. Indeed, exposure to the C-Term peptide significantly decreased the proliferation rate of $C A L R$-mutant MPL-WT-Ba/F3 cells, in the absence of IL-3, relative to untreated controls $(P<$ 0.0001 ) (Figure $4 \mathrm{~A})$. Furthermore, we found that exposure to the peptide resulted in a dose-dependent reduction in JAK/STAT signaling as well as an expansion in cleaved caspase-3 proportions coupled with increased apoptosis rates in treated cells compared with untreated controls $(P<0.05)$ (Figure 4, B and C). Concordantly, we observed a significant loss of MPL expression at the cell surface of MPL-WT-Ba/F3 cells over time after treatment of both CALR DEL- and CALR INS-expressing cells compared with untreated controls (Figure $4 \mathrm{D}$ and supplemental material). To confirm the specificity of this peptide, we designed its inactive analog by replacing all the lysine residues in the C-Term peptide with alanine (KA-mutant peptide). As expected, exposure to the KA-mutant peptide did not influence IL-3-independent growth of CALR-mutant cells and did not decrease JAK/STAT signaling in these cells (Supplemental Figure 4, E and F).

We next sought to delineate the mechanism(s) by which the C-Term peptide attenuated the survival of $C A L R$-mutant cells. By co-immunoprecipitation, we evaluated the effect of C-Term peptide therapy on CALR mutant binding to MPL in cells expressing MPL-WT and MBP-tagged $C A L R$-mutant constructs. We found that C-Term peptide treatment induced a specific disruption of the interaction between mutant CALR and MPL (Figure 4, E and F) and a concurrent reduction in CALR mutant expression levels compared with untreated cells (Figure 4G). Interestingly, in CALR DEL-expressing cells, C-Term peptide treatment was correlated with decreased expression and binding to Fli1, which was not observed in WT CALR-expressing cells (Supplemental Figure 4, G and $\mathrm{H}$ ). To assess whether the peptide could disrupt the chaperone function of mutant CALR proteins, we performed a Western blot analysis on subcellular protein extracts isolated from MPL-WT-Ba/F3 cells expressing CALR mutants after treatment with the C-Term peptide. We observed a decrease in Fli1 protein expression in the chromatin fraction and to a lesser extent in the cytoplasmic compartments of $C A L R$-mutant cells that were treated with the peptide. Interestingly, we also found increased expression of $\mathrm{Mpl}$ and $\mathrm{BiP}$ in the membrane compartment of C-Term-treated cells compared with untreated controls (Figure 4H).

We subsequently investigated whether this peptide could inhibit the proliferation and the MPL/JAK/ STAT signaling in MPN patient cells harboring CALR mutations. We isolated CD $34^{+}$mononuclear cells from patient samples and assessed levels of phosphorylation of STAT3/5 in these cells after treatment with vehicle or the C-Term peptide. We observed that peptide treatment induced a significant decrease in STAT5 
A

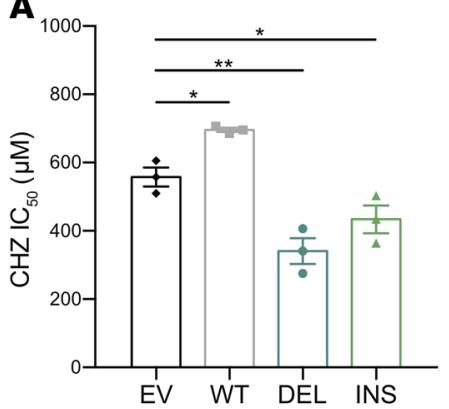

B

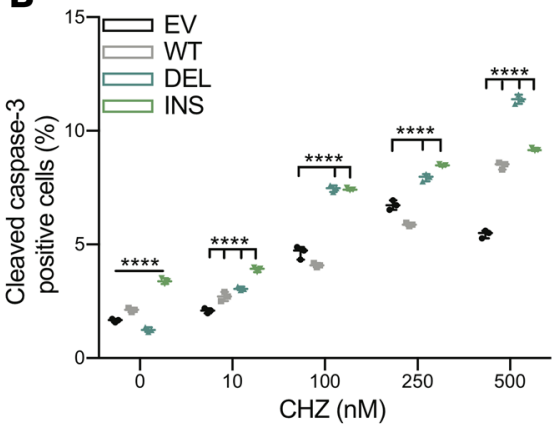

C
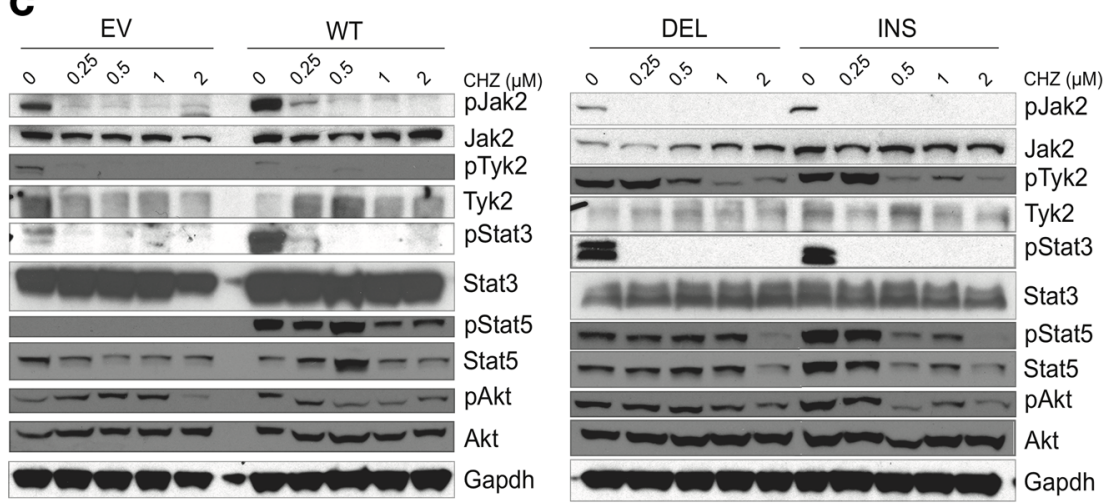

D
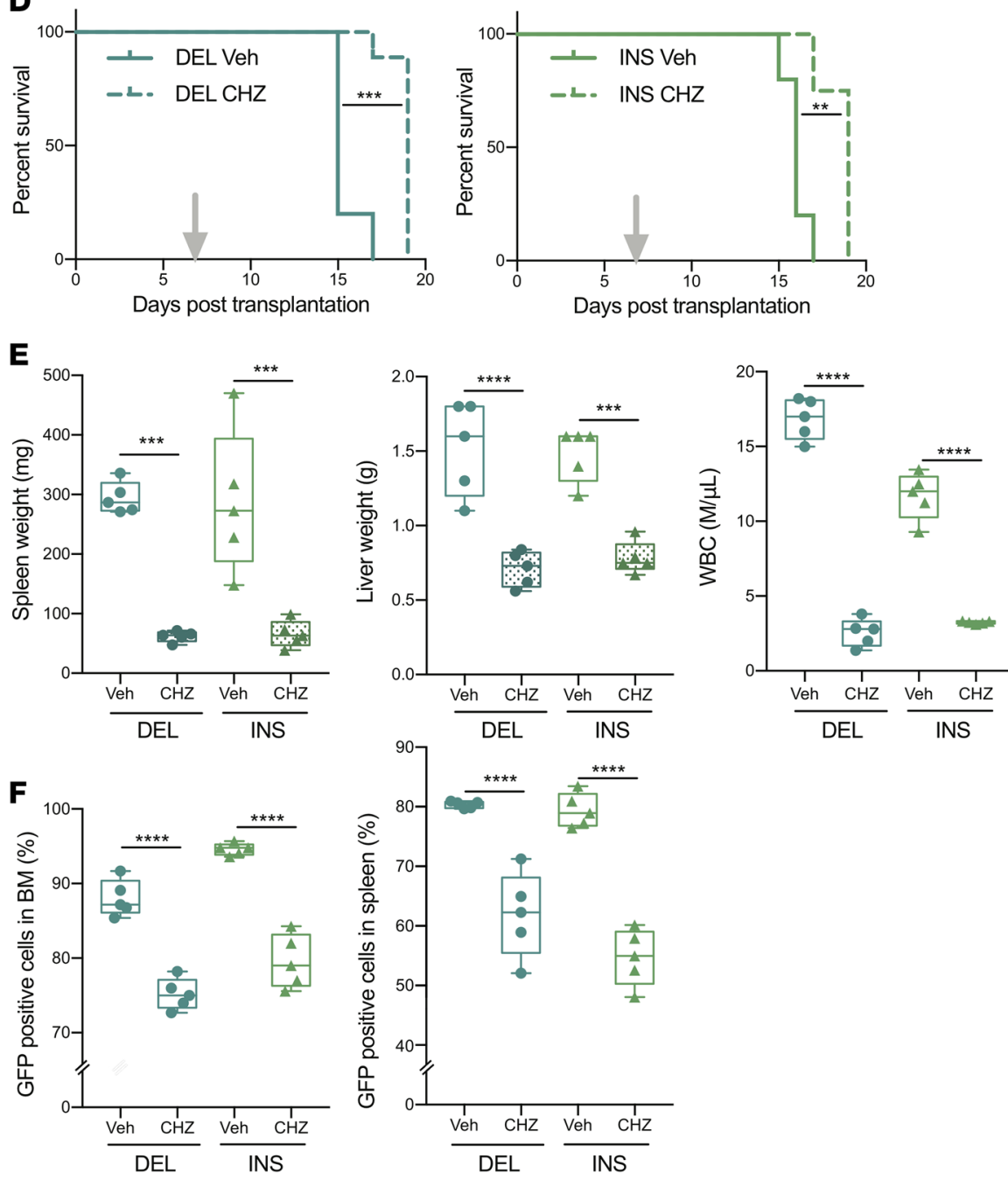

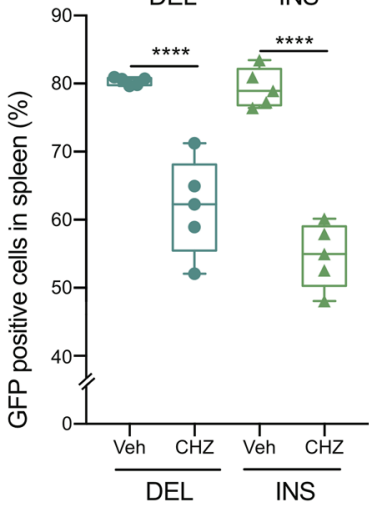

Figure 3. CALR-mutated cells are sensitive to JAK1/2 inhibitors in vitro and in vivo. $(A) I_{50}$ values for MPL-WT-Ba/F3 cells expressing EV or WT, DEL, or INS CALR cultured for 48 hours with increased concentration of $\mathrm{CHZ868}$ ( $\mathrm{CHZ}$, nM) ( $n=3$ in triplicate). (B) Percentage (\%) of cleaved caspase-3-positive MPL-WT-Ba/F3 cells expressing different CALR mutants 24 hours after treatment with increasing concentrations of CHZ868 (CHZ, nM) ( $n=3$ in triplicate). (C) JAK/STAT axis analysis by Western blot analysis on total protein extracts from MPL-WT-Ba/ F3 cells expressing different CALR constructs cultured with increasing concentrations of $\mathrm{CHZ868}(\mathrm{CHZ}, \mu \mathrm{M})$ as compared with negative controls (EV and WT). (D) Kaplan-Meier survival curve, at death, of BALB/c mice injected (arrows) with MPL-WT-Ba/F3 cells expressing DEL CALR or INS CALR and treated with CHZ868. (E) Boxand-whisker plots of spleen weights ( $\mathrm{mg}$ ), liver weights (g), and WBC $(\mathrm{M} / \mu \mathrm{l})$ of DEL CALR- or INS CALR-expressing cells treated with CHZ868. In box-and-whisker plots, horizontal bars indicate the medians, boxes indicate 25th to 75 th percentiles, and whiskers indicate 10th and 90th percentiles. Dots outside of box plots represent outliers (F) Mutant allele burden, as reflected by the percentage of GFP+ cells, in the BM and the spleen, at death of BALB/c mice injected with MPL-WT-Ba/F3 cells expressing DEL CALR or INS CALR and treated with CHZ868 (30 mg/kg). CHZ, CHZ868; veh, vehicle ( $n=10 / 11$ animals per condition). In $\mathbf{A}, \mathbf{B}$, and $\mathbf{D}-\mathbf{F}$, mean values $\pm \mathrm{SEM}$ are represented. Statistical significance was assessed using (A, B, E, and F) 1-way ANOVA or (D) Mantel-Cox; ${ }^{*} P<0.05,{ }^{* *} P<0.01,{ }^{* *} P<$ 0.001 , and ${ }^{* * * *} P<0.0001$. Blots are representative of 3 independent experiments. 
A

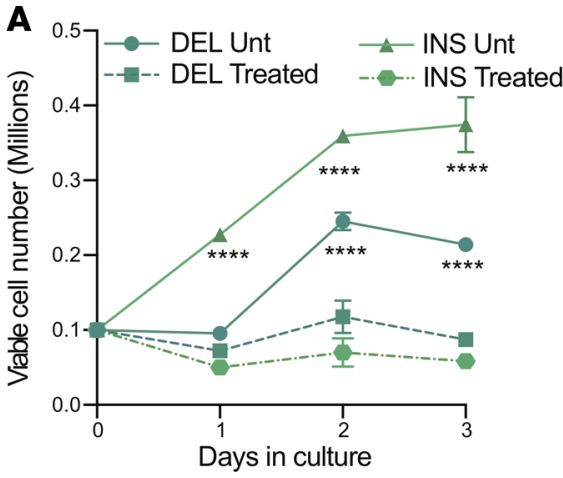

B

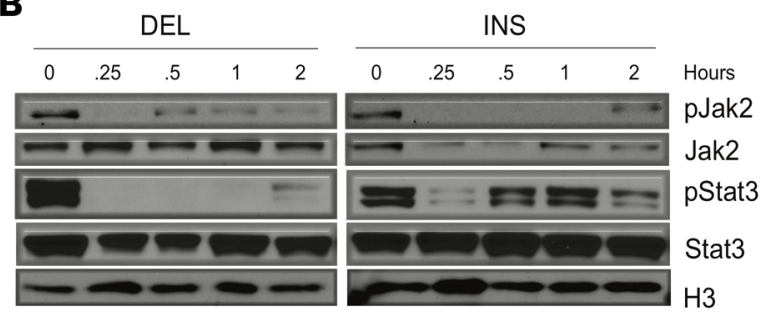

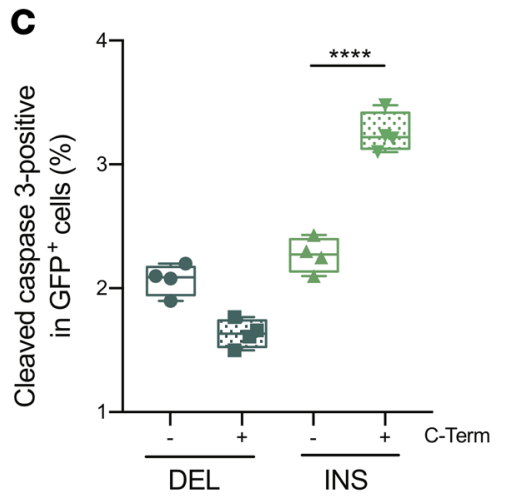

E
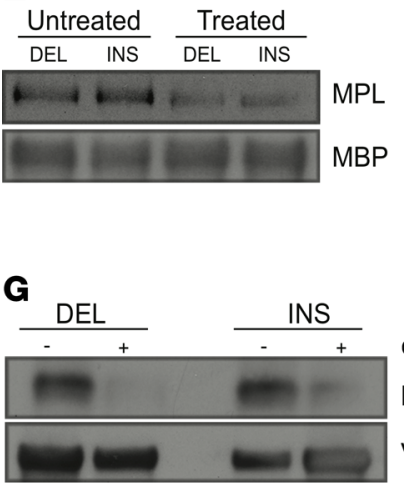

VINCULIN

D

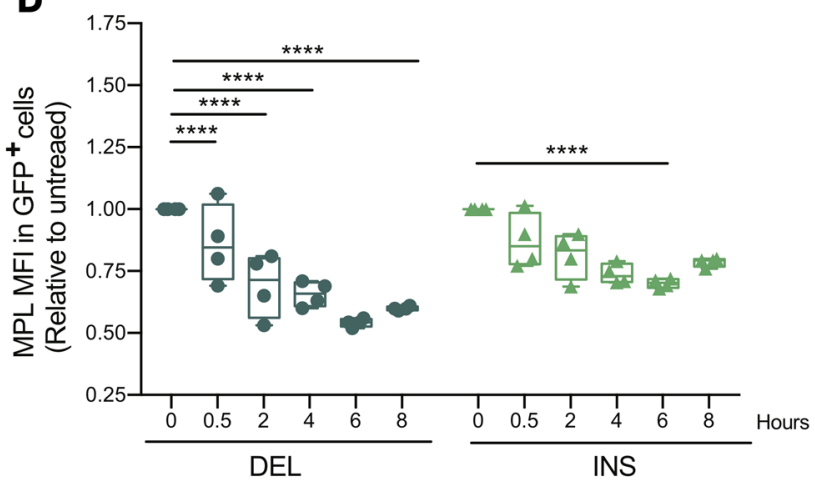

$\mathbf{F}$
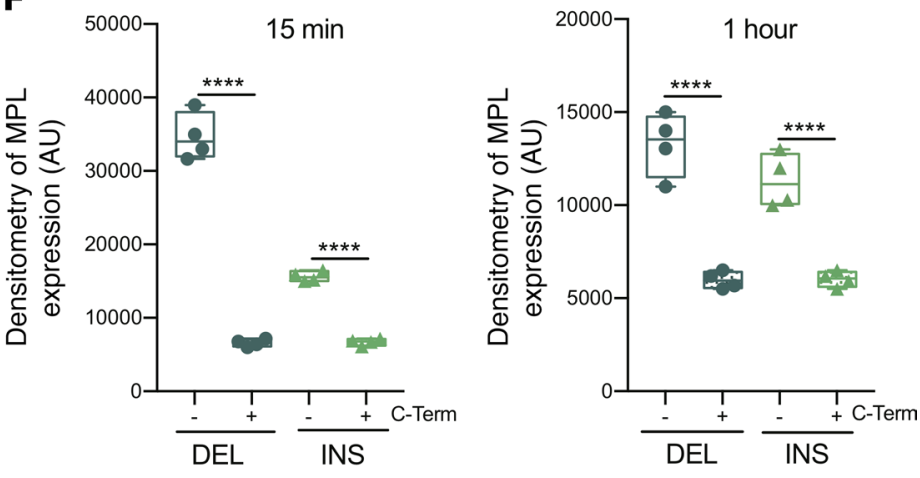

H
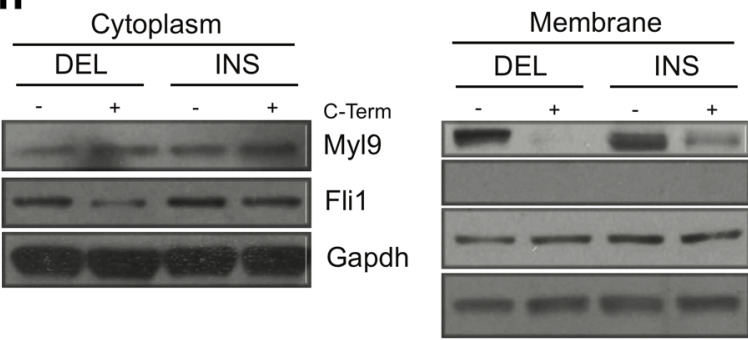

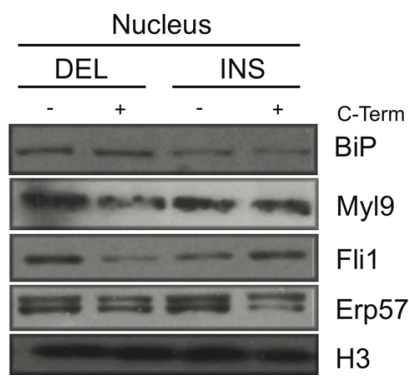

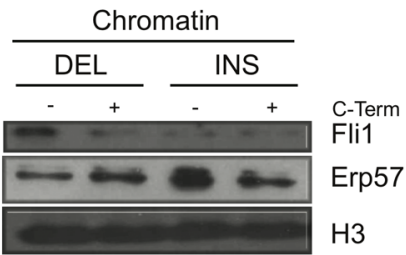

$\mathrm{H} 3$

Figure 4. CALR-mutant cells are sensitive to a peptide corresponding to the C-terminal sequence of the WT CALR. (A) Proliferation assays of MPL-WT-Ba/ F3 cells expressing CALR mutant constructs treated with the C-Term peptide in the absence of IL-3 compared with untreated controls (Unt). Growth curves are means (in total number of viable cells) \pm SEM ( $n=6$ in triplicate). (B) JAK/STAT axis analysis by Western blot analysis of MPL-WT-Ba/F3-cells expressing DEL or INS CALR treated with the C-Term peptide for different incubation times (0-2 hours). (C) Percentage (\%) of cleaved caspase-3-positive MPL-WT-Ba/F3 cells expressing CALR mutants after treatment with the C-Term peptide for 4 hours ( $n=3$ in triplicate). In box-and-whisker plots, horizontal bars indicate the medians, boxes indicate 25 th to 75 th percentiles, and whiskers indicate 10 th and 90 th percentiles. Dots outside of box plots represent outliers. (D) Quantification of change in MPL mean fluorescence index (MFI) in MPL-WT-Ba/F3 cells expressing DEL or INS CALR treated with the C-Term peptide for the indicated time points. (E) Co-immunoprecipitation analysis and (F) quantitative densitometry (AU) of MPL protein expression in 293T cells cotransfected with MPL-WT and MBP-tagged CALR-mutant constructs treated with the peptide for 15 minutes or 1 hour. (C) Western blot analysis of MBP expression in $293 T$ cells transfected 
with MPL-WT and MBP-tagged CALR-mutant constructs and treated with the C-Term peptide for 1 hour. (H) Cytoplasm/membrane and nucleus/chromatin extracts from EV or WT, DEL, or INS CALR-expressing MPL-WT-Ba/F3 cells were used to detect, by Western blot, cellular localization of CALR partners BiP, Myl9, Fli1, and Erp57 after C-Term treatment for 1 hour. In A, C, D, and F, mean values \pm SEM are represented. Statistical significance was assessed using 2-way ANOVA, ${ }^{* * *} P<0.0001$. Blots are representative of 3 independent experiments. Gapdh, histone $\mathrm{H} 3$, and vinculin were used as loading controls.

phosphorylation (pSTAT5) $(P<0.01)$ associated with reduced CALR protein expression (Figure 5A). Likewise, we also observed reduced MPL cell surface expression after treatment (Figure 5B). By contrast, peptide treatment did not influence MPL surface expression in normal hematopoietic cells from healthy donors (CTRL), suggesting that the phenotype is specific to CALR-mutated cells (Supplemental Figure 4I). These results suggest that disruption of interactions between mutant CALR and its chaperone complexes can inhibit the growth and signaling of $C A L R$-mutant cells.

We subsequently determined whether treatment of $C A L R$-mutant cells with the C-Term peptide could improve their sensitivity to JAK1/2 inhibitors. To this end, we treated MPL-WT-Ba/F3 cells expressing EV or WT or mutant CALR with Rux and CHZ alone or in combination with the C-Term peptide. Combination therapy potently abrogated phosphorylation of Stat5 and Akt (pStat5 and pAkt) to a greater extent than JAK1/2 inhibitor treatment alone (Figure 5, C and D). Combined treatment was also more effective at inhibiting the IL-3-independent proliferation of $C A L R$-mutated MPL-WT-Ba/F3 cells compared with $\mathrm{JAK} 1 / 2$ inhibitor treatment alone $\left(\mathrm{IC}_{50}\right.$ for treatment with Rux alone was $0.33 \mu \mathrm{M}$ versus $0.22 \mu \mathrm{M}$ plus C-Term $[P<0.05]$; with $\mathrm{CHZ}$ alone was $0.57 \mu \mathrm{M}$ versus $0.38 \mu \mathrm{M}$ plus $\mathrm{C}$-Term $[P<0.001]$ ) (Figure 5 , E and F). All together, these results suggest that a combination of JAK1/2 inhibitors and the C-Term peptide can improve JAK1/2 inhibitors' potency in vitro.

\section{Discussion}

The identification of recurrent mutations in $C A L R$ in MPN was a pioneering, unexpected finding with respect to gene discovery in patients with myeloid malignancies. CALR, an ER chaperone protein that normally functions to bind misfolded proteins in the ER and prevent their export to the Golgi apparatus, has never previously been reported, to our knowledge, to be mutated in cancer or to be associated with hematologic disorders. Here, we performed functional analysis of mutant CALR proteins and identified protein partners of CALR in $C A L R$-mutated cells. We showed that $C A L R$ mutations modulate differential binding to known partners and allow for interaction with possibly novel partners, including proteins involved in transcriptional regulation (Fli1), ER function (Erp57), unfolded protein response (BiP and Grp75), and the cytoskeleton (My19).

Consistent with the role of CALR as a chaperone and in nuclear import and export (14), we found that CALR mutants altered the cellular localization of a subset of its binding partners (My19 and Fli1), which can result in altered transcriptional regulation of the key CALR effector MPL. We discovered that CALR mutants modulate the expression and cellular localization of Fli1, a known regulator of $M P L$ transcription. In addition, $C A L R$ mutations promote the ability of CALR itself and other CALR-interacting proteins to bind to the $M P L$ promoter. These findings provide insight into how CALR mutants lead to increased MPL/ JAK2 activation through altered epigenetic regulation and inform likely novel mechanisms contributing to CALR-mutant MPN pathogenesis.

Stem cell transplantation is currently the only definitive treatment for MF; however, attendant toxicities and advanced age of MF patients preclude the use of transplantation for most patients. Thus, there remains a compelling need for efficacious drugs for MPN patients, including CALR-mutant MPNs. Rux is a JAK inhibitor that ameliorates splenomegaly and constitutional symptoms associated with $\operatorname{MF}(23,27)$. We observed potent suppression of JAK signaling, and proliferation and induction of apoptosis in CALR-mutant leukemic cell lines treated with either type I (Rux) or type II (CHZ868) JAK2 inhibitors in vitro. Furthermore, we showed that type II JAK2 inhibition with CHZ868 could reduce disease burden in vivo. More importantly, we demonstrated an alternative therapeutic approach by which interference with the mutant CALR interactome can induce therapeutic efficacy. We found that our molecular mimicry of the C-terminal sequence of WT CALR can attenuate IL-3 independency, JAK signaling, and MPL cell surface expression; increase apoptosis of $C A L R$-mutated cell lines and primary samples; and improve the JAK inhibitors' efficacy. Although subsequent preclinical and clinical studies are needed to optimize this approach for in vivo therapeutic delivery, these data inform a possibly novel mechanism by which disruption of the mutant CALR chaperone complexes can be used to develop mechanism-based therapies for patients with $C A L R$-mutant MPN. 

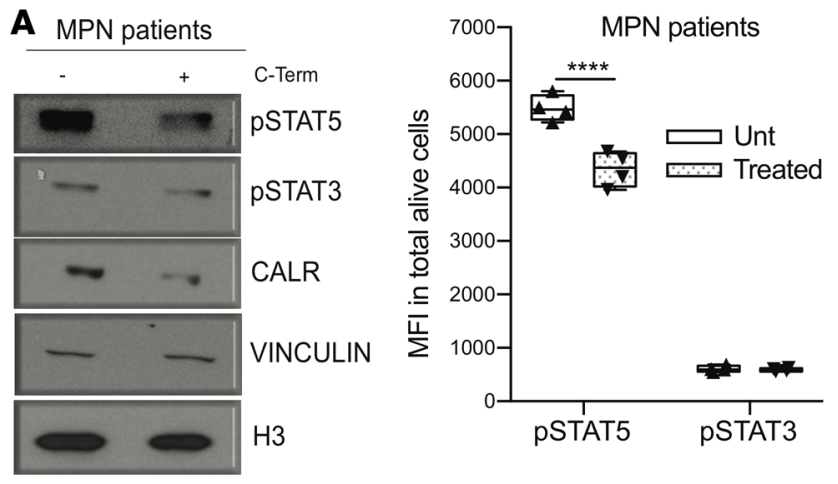

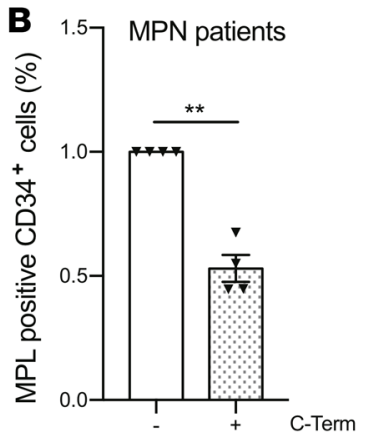

C
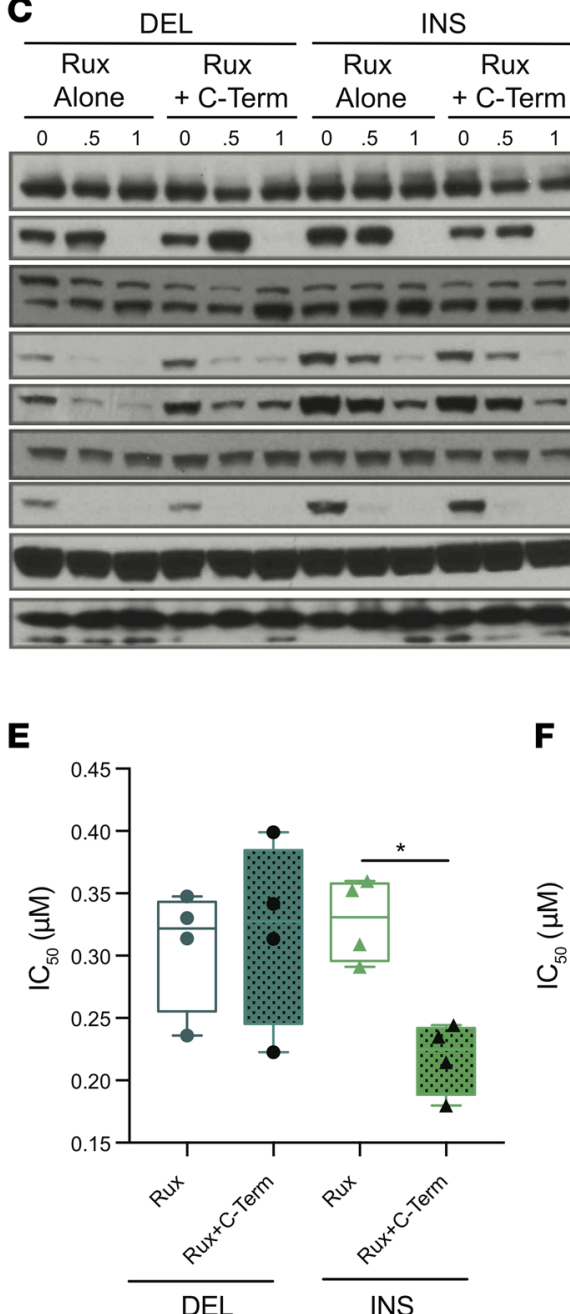

$\mathbf{F}$

MM
MPL
pJak2
Jak2
pStat5 (L)
pStat5 (D)
Stat5
pAkt
Akt
H3

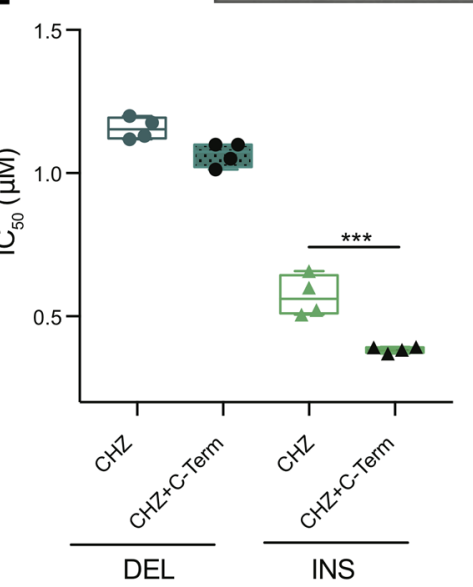

D

\begin{tabular}{|c|c|c|c|}
\hline \multicolumn{2}{|c|}{ DEL } & \multicolumn{2}{|c|}{ INS } \\
\hline $\begin{array}{l}\mathrm{CHZ} \\
\text { Alone }\end{array}$ & $\begin{aligned} & \mathrm{CHZ} \\
+ & \mathrm{C}-\text { Term }\end{aligned}$ & $\begin{array}{l}\mathrm{CHZ} \\
\text { Alone }\end{array}$ & $\begin{aligned} & \mathrm{CHZ} \\
+ & \mathrm{C}-\mathrm{Term}\end{aligned}$ \\
\hline
\end{tabular}

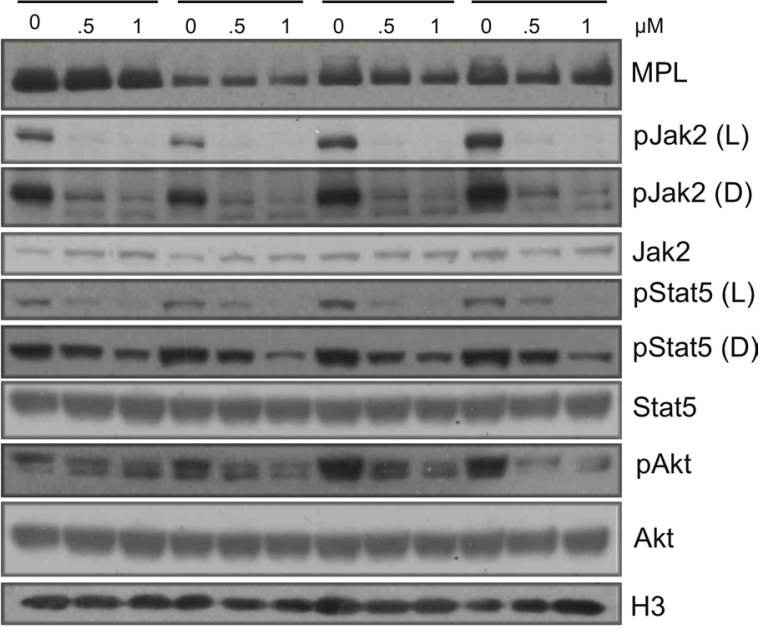

Figure 5. The C-Term peptide represents a tractable approach to target CALR-mutated MPN cells and improve JAK1/2 inhibitors' potency in vitro. (A) Quantification of STAT3/5 phosphorylation levels, by Western blot analysis and flow cytometry, in CD34+ cells isolated from patients with MPN harboring CALR mutations, treated with the C-Term peptide for 1 hour. (B) Quantification of change in MPL-positive cells (\%) among CD34+ cells isolated from patients with CALR-mutated MPN treated with the C-Term peptide for 1 hour. JAK/STAT axis analysis by Western blot of MPL-WT$\mathrm{Ba}$ /F3-cells expressing DEL or INS CALR treated with increasing concentrations $(0-1 \mu \mathrm{M})$ of (C) Rux or (D) CHZ868 (CHZ) alone or in combination with the C-Term peptide for 4 hours. L, light exposure time; D, dark exposure time. $\mathrm{IC}_{50}$ values for MPL-WT-Ba/F3 cells expressing DEL or INS CALR cultured for 48 hours with increasing concentrations of (E) Rux ( $\mu \mathrm{M})$ or $(\mathbf{F}) \mathrm{CHZ}(\mu \mathrm{M})$ alone or in combination with the C-Term peptide $(n=3$ in triplicate). In box-and-whisker plots, horizontal bars indicate the medians, boxes indicate 25th to 75th percentiles, and whiskers indicate 10th and 90th percentiles. Dots outside of box plots represent outliers. In A, B, E, and F, mean values \pm SEM are represented. Statistical significance was assessed using 2-way ANOVA; ${ }^{*} P<0.05,{ }^{* *} P<0.01,{ }^{* *} P<0.001$, and ${ }^{* * *} P<0.0001$. Blots are representative of 3 independent experiments. Histone $\mathrm{H} 3$ was used as a loading control. 


\section{Methods}

Primary cell and cell line cultures. HEK293T (293T), Ba/F3, and UT-7 cell lines were all purchased at American Type Culture Collection and maintained respectively in DMEM, RPMI1640, and Iscove's modified Dulbecco's medium (IMDM) media supplemented with 10\% FBS and 1\% penicillin/streptomycin. All cells were screened for Mycoplasma species by PCR according to the manufacturer's instructions (ABM Inc). Ba/F3 and UT-7 media were supplemented with $10 \mathrm{ng} / \mathrm{ml}$ of murine recombinant IL-3 (PeproTech) and $10 \mathrm{ng} / \mathrm{ml}$ of rhGM-CSF (R\&D Systems), respectively. C-kit ${ }^{+}$cells were isolated from WT C57BL/6 mice using immunomagnetic beads (Miltenyi Biotec, 130-091-224). BM mononuclear cells from BM aspirate from healthy donors or patients with MPN were freshly extracted using Ficoll-Hypaque technique, and $\mathrm{CD} 4^{+}$cells were isolated using immunomagnetic beads (Miltenyi Biotec, 130-046-702). The institutional review board of Memorial Sloan Kettering Cancer Center approved the sample collection and experiments. Informed consent was obtained from all patients providing material for studies. Clinical information about patient samples is in Supplemental Table 1.

Generation of CALR mutant-expressing cell lines. cDNA sequences encoding WT, 52-base pair deletions (DEL), 5-base pair insertions (INS), and STOP (stop codon in exon 9) CALR with a N-terminal MBP tag were cloned into the pMSCV retroviral plasmid with an IRES-GFP. Transient cotransfection of HEK293T cells and generation of retroviral supernatant were performed using Fugene (Roche) according to the manufacturer's guidelines. Human and murine cells were cotransduced with viral supernatants containing MSCV-CALR-IRES-GFP or MSCV-MPLWT-IRES-hCD4 plasmids $(28,29)$; GFP and CD4 ${ }^{+}$cells were FACS sorted using ARIA III (BD Biosciences).

Quantitative reverse transcription $P C R$ and chromatin immunoprecipitation. Total RNA was isolated from the cells using RNeasy Mini Kit (Qiagen) and converted into cDNA by using the Verso cDNA Synthesis Kit (Thermo Fisher Scientific). qRT-PCR was carried out by using SYBR green PCR master mix (Applied Biosystems) (List of primers in Supplemental Table 2). Data were further analyzed by using the comparative $\triangle \mathrm{Ct}$ method. ChIP qPCRs were performed using ChIP-IT Express High-Sensitivity kit (Active Motif), following the manufacturer's instructions. Briefly, cells were fixed and cross-linked with $37 \%$ formaldehyde, then lysed, and DNA was sheared by sonication. Overnight incubations with primary antibodies (FLI1: Abcam ab15289; Erp57: CST 2881; and CALR: CST) were followed by a reverse cross-linking and proteinase $\mathrm{K}$ digestion. The eluted DNA was then processed and analyzed by qRT-PCR. All primers used are listed in Supplemental Table 2 (murine cells: region -191 to $-45 \mathrm{bp}$; human cells: -714 to $-505 \mathrm{bp}$.

Fli1 and Erp57 knock down by retroviral delivery of shRNA. Ba/F3 cells were transduced as previously described (30) with retroviruses expressing puromycin and shRNA-Erp57, shRNA-Fli1 (OriGene), or shRNA-Renilla as control. Cells were infected twice in IL-3-containing media, followed by puromycin selection $(2 \mu \mathrm{g} / \mathrm{ml}, 3-6$ days).

Fli1 ChIP-seq. Briefly, MPL-WT-Ba/F3 cells were cross-linked in 1\% formaldehyde, lysed, and sonicated (COVARIS) to obtain chromatin fragments in a size range between 200 and $700 \mathrm{bp}$. Solubilized chromatin was incubated with Fli1 antibody (Abcam) overnight at $4^{\circ} \mathrm{C}$. Protein A and protein G dynabeads (Thermo Fisher Scientific) were used to capture the antibody/chromatin complex and washed with mixed micelle wash buffer $(150 \mu \mathrm{M} \mathrm{NaCl}, 20 \mu \mathrm{M}$ Tris- $\mathrm{HCl} \mathrm{pH} 8.1,5 \mu \mathrm{M}$ EDTA, 5\% sucrose, $0.02 \% \mathrm{NaN}_{3}, 1 \%$ Triton, $0.2 \%$ SDS); b500 wash buffer ( $0.1 \%$ deoxycholic acid, $1 \mu \mathrm{M}$ EDTA, $50 \mu \mathrm{M}$ HEPES $\mathrm{pH} 7.5,1 \%$ Triton, $\left.0.2 \% \mathrm{NaN}_{3}\right) ; \mathrm{LiCl}$ wash buffer $(0.5 \%$ deoxycholic acid, $1 \mu \mathrm{M}$ EDTA, $250 \mu \mathrm{M} \mathrm{LiCl}, 0.5 \% \mathrm{NP}-40,10 \mu \mathrm{M}$ Tris- $\mathrm{HCl} \mathrm{pH}$ 8.0, 0.2\% NaN $)_{3}$; and TBS wash buffer $(20 \mu \mathrm{M}$ Tris-HCl $\mathrm{pH} 7.4,150 \mu \mathrm{M} \mathrm{NaCl})$. Enriched chromatin fragments were eluted at room temperature for 15 minutes and subjected to cross-link reversal at $65^{\circ} \mathrm{C}$ overnight, before purification with AMPure XP beads (New England BioLabs). ChIP DNA were then quantified by Quant-iT PicoGreen dsDNA Assay Kit (Invitrogen), and libraries were prepared according to the manufacturer's instructions (New England BioLabs ChIP-seq Library Prep kit). The pair-ended FASTQ files were trimmed using TrimGalore and cutadapt and aligned to $\mathrm{mm} 9$ using Bowtie2 (31). Peaks were called using MACS2 with -nomodel parameters and filtering for peaks with a $P \leq 0.001$ (32). Matched input DNA was used as background for the peak calling. Peaks within ENCODE blacklist regions were removed, and all remaining Fli1 peaks per samples were combined into a union peak atlas. Peaks falling within $500 \mathrm{bp}$ from each other were merged. Reads were counted to the peak atlas for each sample using featureCount (33). The summarized peak atlas was normalized using DEseq. 2 (33) based on all reads mapping to the peaks. Using the normalization factors from DEseq. 2, normalized bigWig files were created using bedtools genomeCoverageBed (34). 
ChIP reads were extended with $200 \mathrm{bp}$ for smoothing. Bwtools (35) was used to calculate the normalized reads $\pm 5 \mathrm{~kb}$ around each peak. Homer's (36) annotatePeaks.pl was used to annotate the genic location of all individual peak calls per sample. All original ChIP-seq data were deposited in the NCBI's Gene Expression Omnibus database (GEO GSE120134).

In vitro treatment with C-Term peptide or inhibitors. Transduced 293T, MPL-WT-Ba/F3, as well as CD $34^{+}$cells were treated with $20 \mu \mathrm{M}$ of C-Term WT CALR (Myr-KEDDEDKDEDEEDEEDKEEDEEEDVPGQAKDEL-OH), Biotag peptide (Myr-KEDDEDKDEDEEDEED(KLCBiot)EEDEEEDVPGQAKDEL-OH), or KA-mutant peptides (Myr-AEDDEDADEDEEDEEDAEEDEEEDVPGQAADEL-OH) (New England Peptide) for different time points (0 to 8 hours) using ECB buffer as previously described (37). To increase peptide uptake by hematopoietic cells and improve its stability, a myristoylated lysine was added to the N-terminus of the WT CALR peptide (37).

Statistics and data availability. Sample sizes were not predetermined and the experiments were not randomized. Information on statistical tests used and $P$ values are given in the figure legends, as well as the number of independent repeats of experiments and sample size. All data supporting this study are available from the corresponding author on reasonable request. Mass spectrometry data that support the findings of this study have been deposited via ProteomeXchange repository with accession number PXD008476.

Study approval. Human studies were approved by the institutional review board of the Memorial Sloan Kettering Cancer Center. Informed consent was obtained from all participants before inclusion in the study. All animal protocols were approved by the Memorial Sloan Kettering Cancer Center IACUC. Studies were conducted under guidelines for animal welfare provided by the NIH (Guide for the Care and Use of Laboratory Animals, National Academies Press, 2011).

\section{Author contributions}

EP, RLL, AK, and JL conceived and designed the study. EP, TRM, KBB, AVHS, KEW, JL, and FP performed and designed the experimental procedures. $\mathrm{PC}$ and $\mathrm{AK}$ performed the mass spectrometry experiments and analysis. JLVM, JP, and RK performed and provided help for the ChIP-seq experiments. RKR provided MPN patient samples. EP and RLL wrote the manuscript.

\section{Acknowledgments}

This work was supported by R35 CA197594-01A1 (to RLL), by R01 CA204396 (to AK), by P01 CA108671 (to RLL), by a Specialized Center of Research Program grant from the Leukemia and Lymphoma Society (to RLL), by a grant from Cycle for Survival (to RLL), by the Memorial Sloan Kettering Cancer Center Support Grant (NIH P30 CA008748), and by the François Wallace Monahan Fellowship (to EP). We acknowledge the use of the Integrated Genomics Operation Core, funded by the Memorial Sloan Kettering Cancer Center Support Grant (NIH P30 CA008748) and the Marie-Josée and Henry R. Kravis Center for Molecular Oncology. AK is the Damon Runyon-Richard Lumsden Foundation Clinical Investigator. We would like to thank Robert Bowman for his advice and feedback during the preparation of this manuscript.

Address correspondence to: Ross L. Levine, Human Oncology and Pathogenesis Program, Memorial Sloan Kettering Cancer Center, Box 20, New York, New York 10065, USA. Phone: 646.888.2767; Email: leviner@mskcc.org.

1. Klampfl T, et al. Somatic mutations of calreticulin in myeloproliferative neoplasms. N Engl J Med. 2013;369(25):2379-2390.

2. Nangalia J, et al. Somatic CALR mutations in myeloproliferative neoplasms with nonmutated JAK2. N Engl J Med. 2013;369(25):2391-2405.

3. Elf S, et al. Mutant calreticulin requires both its mutant C-terminus and the thrombopoietin receptor for oncogenic transformation. Cancer Discov. 2016;6(4):368-381.

4. Li J, et al. Mutant calreticulin knock-in mice develop thrombocytosis and myelofibrosis without a stem cell self-renewal advantage. Blood. 2018;131(6):649-661.

5. Marty C, et al. Calreticulin mutants in mice induce an MPL-dependent thrombocytosis with frequent progression to myelofibrosis. Blood. 2016;127(10):1317-1324.

6. Shide $\mathrm{K}$, et al. Calreticulin mutant mice develop essential thrombocythemia that is ameliorated by the JAK inhibitor ruxolitinib. Leukemia. 2017;31(5):1136-1144.

7. Araki M, et al. Activation of the thrombopoietin receptor by mutant calreticulin in CALR-mutant myeloproliferative neoplasms. Blood. 2016;127(10):1307-1316.

8. Chachoua I, et al. Thrombopoietin receptor activation by myeloproliferative neoplasm associated calreticulin mutants. Blood. 
2016;127(10):1325-1335.

9. Fox JD, Routzahn KM, Bucher MH, Waugh DS. Maltodextrin-binding proteins from diverse bacteria and archaea are potent solubility enhancers. FEBS Lett. 2003;537(1-3):53-57.

10. Garbati MR, et al. Cytokine overproduction and crosslinker hypersensitivity are unlinked in Fanconi anemia macrophages. J Leukoc Biol. 2016;99(3):455-465.

11. Kollmann $\mathrm{K}$, et al. A novel signalling screen demonstrates that CALR mutations activate essential MAPK signalling and facilitate megakaryocyte differentiation. Leukemia. 2017;31(4):934-944.

12. Elf S, et al. Defining the requirements for the pathogenic interaction between mutant calreticulin and MPL in MPN. Blood. 2018;131(7):782-786.

13. Roderick HL, Campbell AK, Llewellyn DH. Nuclear localisation of calreticulin in vivo is enhanced by its interaction with glucocorticoid receptors. FEBS Lett. 1997;405(2):181-185.

14. Holaska JM, Black BE, Love DC, Hanover JA, Leszyk J, Paschal BM. Calreticulin is a receptor for nuclear export. J Cell Biol. 2001;152(1):127-140.

15. Deveaux S, Filipe A, Lemarchandel V, Ghysdael J, Romeo PH, Mignotte V. Analysis of the thrombopoietin receptor (MPL) promoter implicates GATA and Ets proteins in the coregulation of megakaryocyte-specific genes. Blood. 1996;87(11):4678-4685.

16. Ndubuisi MI, Guo GG, Fried VA, Etlinger JD, Sehgal PB. Cellular physiology of STAT3: Where's the cytoplasmic monomer? J Biol Chem. 1999;274(36):25499-25509.

17. Altieri F, Maras B, Eufemi M, Ferraro A, Turano C. Purification of a 57kDa nuclear matrix protein associated with thiol:protein-disulfide oxidoreductase and phospholipase C activities. Biochem Biophys Res Commun. 1993;194(3):992-1000.

18. Gerner C, Holzmann K, Meissner M, Gotzmann J, Grimm R, Sauermann G. Reassembling proteins and chaperones in human nuclear matrix protein fractions. J Cell Biochem. 1999;74(2):145-151.

19. Ohtani H, Wakui H, Ishino T, Komatsuda A, Miura AB. An isoform of protein disulfide isomerase is expressed in the developing acrosome of spermatids during rat spermiogenesis and is transported into the nucleus of mature spermatids and epididymal spermatozoa. Histochemistry. 1993;100(6):423-429.

20. Dedhar S, et al. Inhibition of nuclear hormone receptor activity by calreticulin. Nature. 1994;367(6462):480-483.

21. Jackers P, Szalai G, Moussa O, Watson DK. Ets-dependent regulation of target gene expression during megakaryopoiesis. J Biol Chem. 2004;279(50):52183-52190.

22. Huang H, et al. Differentiation-dependent interactions between RUNX-1 and FLI-1 during megakaryocyte development. Mol Cell Biol. 2009;29(15):4103-4115.

23. Harrison C, et al. JAK inhibition with ruxolitinib versus best available therapy for myelofibrosis. NEngl J Med. 2012;366(9):787-798

24. Verstovsek S, et al. Long-term outcomes of 107 patients with myelofibrosis receiving JAK1/JAK2 inhibitor ruxolitinib: survival advantage in comparison to matched historical controls. Blood. 2012;120(6):1202-1209.

25. Koppikar P, et al. Heterodimeric JAK-STAT activation as a mechanism of persistence to JAK2 inhibitor therapy. Nature. 2012;489(7414):155-159.

26. Meyer SC, et al. CHZ868, a type II JAK2 inhibitor, reverses type I JAK inhibitor persistence and demonstrates efficacy in myeloproliferative neoplasms. Cancer Cell. 2015;28(1):15-28.

27. Verstovsek S, et al. A double-blind, placebo-controlled trial of ruxolitinib for myelofibrosis. N Engl J Med. 2012;366(9):79-807.

28. Pikman Y, et al. MPLW515L is a novel somatic activating mutation in myelofibrosis with myeloid metaplasia. PLoS Med. 2006;3(7):e270.

29. Artinger EL, et al. An MLL-dependent network sustains hematopoiesis. Proc Natl Acad Sci U S A. 2013;110(29):12000-12005.

30. Kunimoto $\mathrm{H}$, et al. Cooperative epigenetic remodeling by TET2 loss and NRAS mutation drives myeloid transformation and MEK inhibitor sensitivity. Cancer Cell. 2018;33(1):44-59.e8.

31. Martin M. Cutadapt removes adapter sequences from high-throughput sequencing reads. EMBnet.journal. 2011;17(1):10-12.

32. Zhang Y, et al. Model-based analysis of ChIP-Seq (MACS). Genome Biol. 2008;9(9):R137.

33. Liao Y, Smyth GK, Shi W. featureCounts: an efficient general purpose program for assigning sequence reads to genomic features. Bioinformatics. 2014;30(7):923-930.

34. Quinlan AR, Hall IM. BEDTools: a flexible suite of utilities for comparing genomic features. Bioinformatics. 2010;26(6):841-842.

35. Pohl A, Beato M. bwtool: a tool for bigWig files. Bioinformatics. 2014;30(11):1618-1619.

36. Heinz S, et al. Simple combinations of lineage-determining transcription factors prime cis-regulatory elements required for macrophage and B cell identities. Mol Cell. 2010;38(4):576-589.

37. Nelson AR, Borland L, Allbritton NL, Sims CE. Myristoyl-based transport of peptides into living cells. Biochemistry. 2007;46(51):14771-14781. 